\title{
Thieme Chemistry Journal Awardees - Where Are They Now? Bifunctional Silver Acetate Catalyzed Asymmetric Mannich-Type Reactions
}

\author{
Qing-An Chen, Wei Zeng, Da-Wei Wang, Yong-Gui Zhou* \\ State Key Laboratory of Catalysis, Dalian Institute of Chemical Physics, Chinese Academy of Sciences, \\ 457 Zhongshan Road, Dalian 116023, P. R. of China \\ Fax +86(411)84379220; E-mail: ygzhou@dicp.ac.cn \\ Received 5 June 2009
}

\begin{abstract}
Efficient bifunctional silver acetate catalyzed three asymmetric Mannich-type reactions with high reactivity, up to $>95: 5$ diastereoselectivities and moderate to excellent enantioselectivities (up to $97 \%$ ee). External base is not required in this bifunctional catalytic procedure, in which acetate promotes the deprotonation.
\end{abstract}

Key words: bifunctional, silver acetate, Mannich reaction, asymmetric catalysis, imines

The asymmetric Mannich reaction is one of the most powerful and widely utilized chemical transformation for the preparation of optically active $\beta$-amino carbonyl compounds and related compounds, ${ }^{1}$ which are key chiral building blocks found in various natural products and pharmaceutically important compounds. ${ }^{2}$ Therefore, various enantioselective and diastereoselective Mannich-type reactions catalyzed by either organometallic complexes ${ }^{3-7}$ or organocatalysts ${ }^{8-11}$ have been developed over the past decade. However, for the transition-metal-catalyzed asymmetric Mannich reactions, a catalytic or stoichiometric amount of base is usually crucial for achieving full conversion in most cases of these reactions. Thus, developing an efficient asymmetric Mannich reaction without extra base under mild conditions is attractive.

Recently, we demonstrated that bifunctional silver acetate catalyzed asymmetric cycloaddition of azomethine ylides with electron-deficient alkenes using ferrocenyloxazoline-derived N,P-ligands. ${ }^{12}$ The reactive metal-bound azomethine ylide dipole is formed through deprotonation by the acetate, which plays the role of base, meaning that extra base is not necessary. We envisioned that the introduction of an ion with proper basicity to the Lewis acid might be an appealing choice for the enolization of carbonyl compounds, and that the same strategy could be applied to the Lewis acid catalyzed Mannich reaction. Herein, we describe three examples of asymmetric Mannich-type reactions catalyzed by chiral silver acetate complexes, which acts as a bifunctional catalyst, with low to high diastereoselectivities and moderate to excellent enantioselectivities.

Based on such considerations and on other groups' work, ${ }^{13}$ we set out to establish whether the catalytic system depicted in Table 1 could be used to effect catalytic

SYNLETT 2009, No. 14, pp 2236-2241

Advanced online publication: 07.08.2009

DOI: 10.1055/s-0029-1217805; Art ID: S05709ST

(c) Georg Thieme Verlag Stuttgart · New York

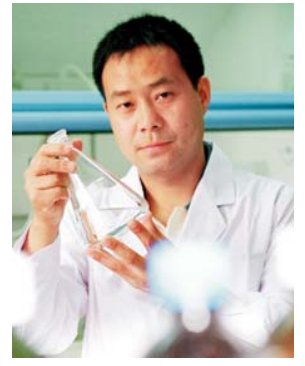

Yong-Gui Zhou was born in 1970 in Hubei Province, China, received a B.Sc. degree from Huaibei Coal Industrial Teachers' College in 1993 and earned his Ph.D. from Shanghai Institute of Organic Chemistry in 1999, working under the direction of Profs. Li-Xin Dai and Xue-Long Hou. He joined Xumu Zhang's group at the Pennsylvania State University as a postdoctoral fellow that same year, and in 2002 he began his independent research career at the Dalian Institute of Chemical Physics, Chinese Academy of Sciences, where currently he is Professor of Chemistry. His research interests include the development of catalytic asymmetric reactions, mechanistic elucidation, and asymmetric synthesis. He has authored over 50 publications and holds over five patents, and received the Thieme Synlett/Synthesis Journals Award in 2006.

asymmetric Mannich reactions between acetyl acetone and $N$-Boc-protected arylimines $\mathbf{1}$.

In our initial investigation, we found that the silver acetate-L1 system can efficiently catalyze the Mannich reaction of aldimines 1a with acetyl acetone in diethyl ether with high activity and moderate enantioselectivity $(67 \%$ ee) in the absence of base (entry 1, Table 1). Encouraged by this result, an evaluation of a range of solvents on the activity and enantioselectivity was carried out (entries 1 4). The results showed that diethyl ether was superior to other solvents.

When ligand $\left(S, S_{\mathrm{P}}\right)-\mathbf{L} \mathbf{2}$, with the same central chirality and opposite planar chirality to $\left(S, R_{\mathrm{P}}\right)-\mathbf{L} \mathbf{1}$, was used in this reaction to investigate the effect of planar chirality on the enantioselectivity and absolute configuration of the products, lower enantiomeric excess (ee) was observed (entry $5)$. The lower ee with $\left(S, S_{\mathrm{P}}\right)$-L2 $\mathbf{2}$ suggests the mismatched nature of the $(S)$-planar chirality with the $(S)$-central chirality on the ligand. Subsequently, the effect of the substituent R in ligands $\mathbf{L 3 a}-\mathbf{L 3 c}$ was examined in order to improve the enantioselectivity. A better enantioselectivity (77\% ee) was obtained with ligand $\mathbf{L 3 b}$ bearing a strong electron-donating group at the para-position of the phenyl on the sulfur atom (entry 7).

The influence of the steric and electronic properties of the substituents of the phosphorus atom in ligand L3b was 
also studied (entries 9-12). The results revealed that the enantioselectivity of this Mannich-type reaction was more sensitive to electronic effects than to the steric properties of the substituents of the phenyl group on the phosphorus atom. The enantioselectivity dramatically decreased when L3g, having a strong electron-withdrawing group at the phenyl ring on the phosphorus atom was used (entry 12). Increasing the steric effect of the ligand slightly improved the reaction enantioselectivity (entries 9-10). A higher ee was obtained at lower reaction temperatures (entries 13-
14). Thus, the optimal conditions for this asymmetric Mannich reaction are $\mathrm{AgOAc} / \mathrm{L3e} / \mathrm{Et}_{2} \mathrm{O} /-40{ }^{\circ} \mathrm{C}$.

Having established the optimal conditions, the scope of the silver acetate catalyzed Mannich reaction was explored (Table 2). In general, good enantioselectivities and high isolated yields were obtained regardless of the electronic properties and steric hindrance of the phenyl ring of aldimines 1 (entries 1-7). A slightly lower enantioselectivity was obtained when the heteroaromatic aldimine $\mathbf{1 h}$ was used (entry 8).

Table 1 Optimization of the Asymmetric Mannich Reaction between Acetyl Acetone and $N$-Boc-Protected Arylimine $\mathbf{1 a}^{25}$

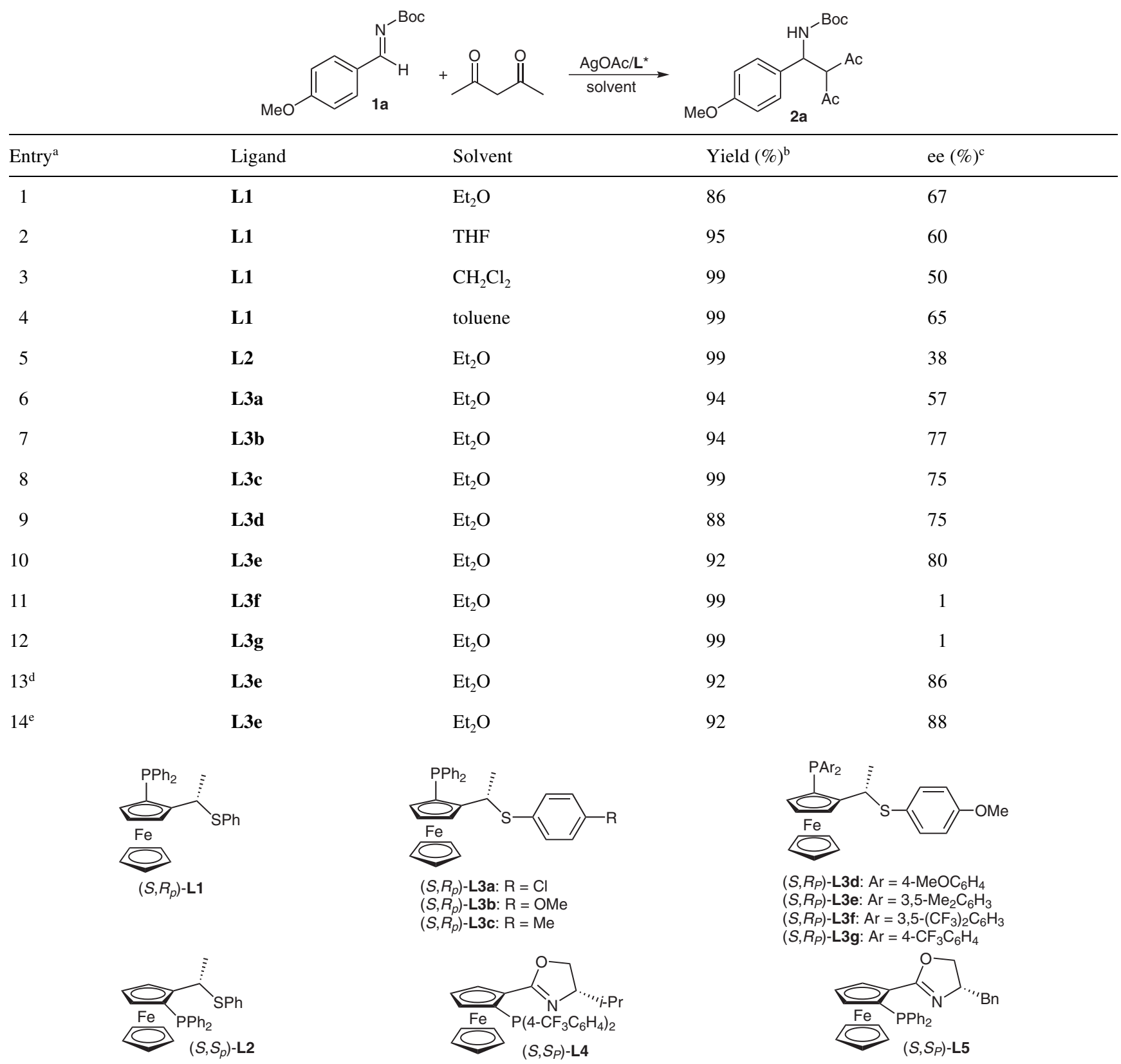

\footnotetext{
${ }^{a}$ Reaction conditions: 1 (1.0 equiv), acetyl acetone (1.2 equiv), $\mathrm{AgOAc}(3 \mathrm{~mol} \%)$, ligand $(3.3 \mathrm{~mol} \%)$, concentration $(0.12 \mathrm{M}), 0{ }^{\circ} \mathrm{C}$.

${ }^{\mathrm{b}}$ Isolated yields based on $\mathbf{1}$.

${ }^{\mathrm{c}}$ Determined by chiral HPLC analysis.

${ }^{\mathrm{d}} \mathrm{At}-25^{\circ} \mathrm{C}$.

${ }^{\mathrm{e}} \mathrm{At}-40^{\circ} \mathrm{C}$.
} 
Table 2 Silver Acetate Catalyzed Asymmetric Mannich Reaction between Acetyl Acetone and $N$-Boc-Protected Arylimines ${ }^{25}$

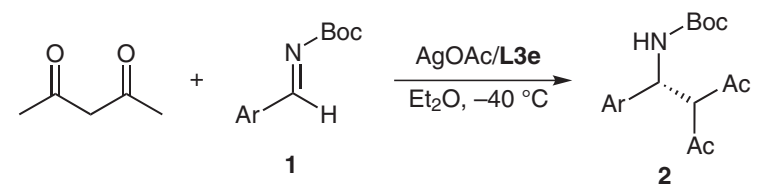

\begin{tabular}{llllll}
\hline Entry $^{\mathrm{a}}$ & $\mathrm{Ar}$ & Product & \multicolumn{2}{l}{ Time (h) } & Yield $(\%)^{\mathrm{b}}$ ee $(\%)^{\mathrm{c}}$ \\
\hline 1 & $4-\mathrm{MeOC}_{6} \mathrm{H}_{4}(\mathbf{1 a})$ & $\mathbf{2 a}$ & 0.5 & 92 & 88 \\
2 & $\mathrm{Ph}(\mathbf{1 b})$ & $\mathbf{2 b}$ & 3 & 67 & $86^{\mathrm{d}}$ \\
3 & $4-\mathrm{MeC}_{6} \mathrm{H}_{4}(\mathbf{1 c})$ & $\mathbf{2 c}$ & 3 & 82 & 88 \\
4 & $4-\mathrm{FC}_{6} \mathrm{H}_{4}(\mathbf{1 d})$ & $\mathbf{2 d}$ & 1 & 99 & 91 \\
5 & $4-\mathrm{BrC}_{6} \mathrm{H}_{4}(\mathbf{1 e})$ & $\mathbf{2 e}$ & 2.5 & 81 & 90 \\
6 & $2-\mathrm{MeC}_{6} \mathrm{H}_{4}(\mathbf{1 f})$ & $\mathbf{2 f}$ & 3 & 86 & 88 \\
7 & 1-naphthyl (1g) & $\mathbf{2 g}$ & 5 & 95 & 88 \\
8 & 2-furyl (1h) & $\mathbf{2 h}$ & 5 & 88 & 80 \\
\hline
\end{tabular}

${ }^{\text {a }}$ Reaction conditions: 1 (1.0 equiv), acetyl acetone (1.2 equiv),

$\mathrm{AgOAc}(3 \mathrm{~mol} \%)$, ligand $(3.3 \mathrm{~mol} \%)$, concentration $(0.12 \mathrm{M})$.

${ }^{\mathrm{b}}$ Isolated yields based on $\mathbf{1}$.

${ }^{\mathrm{c}}$ Determined by chiral HPLC analysis.

${ }^{\mathrm{d}}$ The absolute configuration was determined to be $R$ by comparison with literature data. ${ }^{13 a}$

The silver acetate catalyzed Mannich reaction was also applicable to other $\beta$-dicarbonyl compounds (Scheme 1). High isolated yields (86-90\%) and good enantioselectivities $(78 \%$ ee) were obtained for both malonate and $\beta$-keto ester. However, no valuable diastereoselectivity (dr 2:1) was observed in the asymmetric Mannich reaction between $\beta$-keto ester and aldimine $\mathbf{1 b}$, probably due to epimerization. ${ }^{13 \mathrm{k}}$

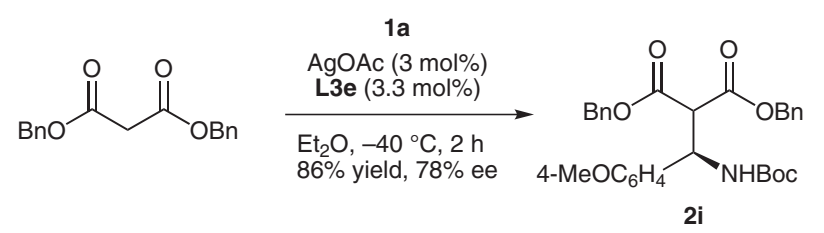

$1 b$
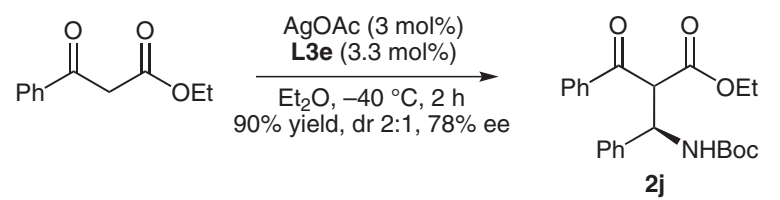

Scheme 1 Silver acetate catalyzed asymmetric Mannich reaction between $\beta$-dicarbonyl compounds and $N$-Boc-protected arylimines

Optically active $\alpha, \beta$-diamino acids represent an important class of compounds critical to a variety of biologically active molecules. ${ }^{2}$ The Mannich-type reaction of glycine Schiff base $\mathbf{3}$ and related compounds with imines, provides an efficient and convenient route for the preparation of these compounds. In 2003, Jørgensen's group ${ }^{14}$ developed the first example of phosphino-oxazoline-copper(I) complex catalyzed Mannich reaction of imino glycine alkyl esters with imines. Subsequently, Maruoka, ${ }^{15}$ Shibasaki, ${ }^{16}$ Kobayashi, ${ }^{17}$ Hou, ${ }^{18}$ Carretero, ${ }^{19}$ and Feng ${ }^{20}$ reported other efficient catalysts for diastereoselective and enantioselective Mannich-type reactions of glycine Schiff bases with aldimines. Encouraged by the success in silver acetate catalyzed direct addition of acetyl acetone to $\mathrm{N}$ Boc-protected arylimines, we decided to develop a bifunctional silver acetate catalyzed enantioselective Mannich reaction of glycine Schiff base with $N$-tosylprotected imine 4 .

Table 3 Silver Acetate Catalyzed Asymmetric Mannich Reaction of Glycine Schiff Bases with Aldimines $\mathbf{4}^{25}$

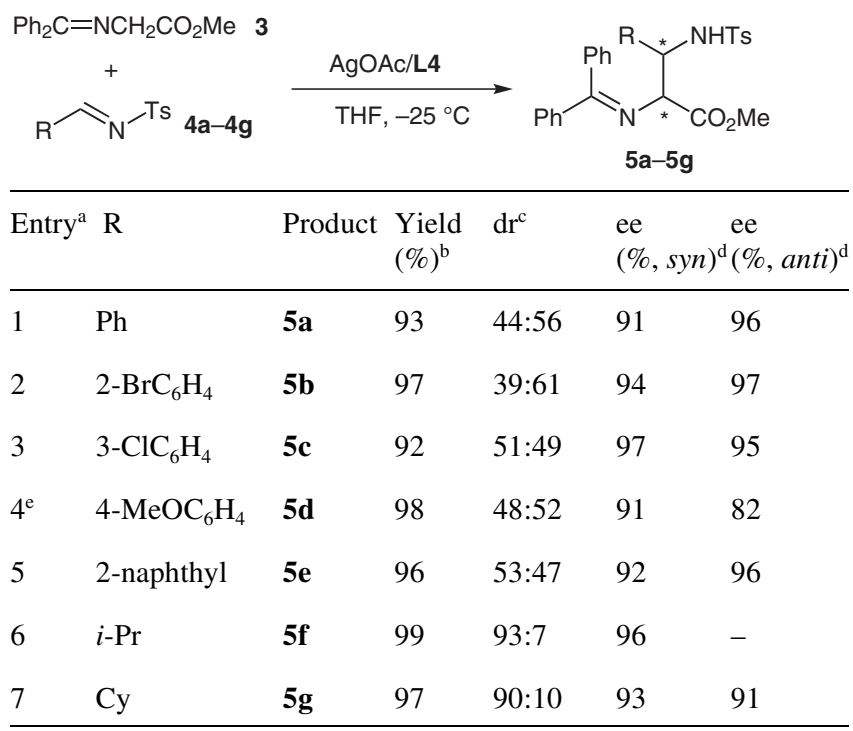

${ }^{\mathrm{a}}$ Reaction conditions: 3 (1.0 equiv), 4 (1.2 equiv), $\mathrm{AgOAc}(3 \mathrm{~mol} \%)$, ligand $(3.3 \mathrm{~mol} \%)$, concentration $(0.12 \mathrm{M}), 3-4 \mathrm{~h}$.

${ }^{\mathrm{b}}$ Isolated yields based on $\mathbf{3}$.

${ }^{\mathrm{c}} \mathrm{dr}=$ syn/anti, determined by ${ }^{1} \mathrm{H}$ NMR.

${ }^{\mathrm{d}}$ Determined by chiral HPLC analysis.

e $22 \mathrm{~h}$.

We initially focused on the exploration of appropriate chiral ligands and on an examination of reaction parameters, such as solvent, temperature, and concentration. The best results, with respect to reactivity, yield, and selectivity, were obtained with silver acetate in combination with $\mathbf{L 4}$ as the catalyst in THF at $-25^{\circ} \mathrm{C}$.

Under the optimized conditions, a series of aromatic imines with electron-withdrawing and electron-donating substituents underwent the desired transformation to give the corresponding $\alpha, \beta$-diamino acid esters $\mathbf{5}$ with good yields and excellent enantioselectivities for both diastereomers but low diastereoselectivities (entries 1-5, Table 3). To our satisfaction, for the aliphatic imines, excellent enantioselectivities (96\% and $93 \%$ ee) and high diastereoselectivities (up to 93:7) were obtained (entries 6 and 7).

As a variant of the Mannich reaction, the asymmetric vinylogous Mannich (AVM) reaction has gained increasing attention owing to its ability to directly generate complex and highly functionalized $\delta$-amino compounds. ${ }^{\text {dd-f, } 9 \text {-g, }, 21}$ 
Recently, Chen et al..$^{22}$ developed an efficient asymmetric vinylogous Mannich reaction of $\alpha, \alpha$-dicyanoolefins ${ }^{23}$ with $N$-Boc-aldimines catalyzed by a bifunctional thiourea-tertiary amine catalyst. Soon thereafter, Jørgensen's group ${ }^{24}$ presented a stereoselective vinylogous Mannich reaction of dicyanoalkylidenes under phase-transfer catalytic conditions utilizing stable $\alpha$-amido sulfones as imine precursors. In a continuation of our studies on the silver acetate catalyzed enantioselective Mannich reaction and on the basis of our experience in aforementioned work, we decided to examine a bifunctional silver acetate catalyzed asymmetric vinylogous Mannich reaction.

Table 4 Silver Acetate Catalyzed Asymmetric Mannich Reaction of $\alpha, \alpha$-Dicyanoolefins 6 with $N$-Boc-Aldimines $\mathbf{1}^{25}$<smiles>[R]CC([R])=C(C#N)C#N</smiles>

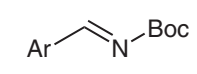
1<smiles>N#CC(C#N)=C1CCCc2ccccc21</smiles>
$6 a$ 6b

\begin{tabular}{llllllll}
\hline Entry $^{\mathrm{a}} \mathbf{6}$ & $\mathrm{Ar}$ & \multicolumn{3}{c}{$\begin{array}{c}\text { Product } \\
\text { Time } \\
(\mathrm{h})\end{array}$} & $\begin{array}{l}\mathrm{Yield}^{\mathrm{b}} \\
(\%)^{\mathrm{b}}\end{array}$ & $\begin{array}{l}\mathrm{ee} \\
(\%)^{\mathrm{d}}\end{array}$ \\
\hline 1 & $\mathbf{6 a}$ & $\mathrm{Ph}(\mathbf{1 b})$ & $\mathbf{7 \mathbf { a } ^ { 2 6 }}$ & 4 & 88 & $93: 7$ & 64 \\
2 & $\mathbf{6 b}$ & $\mathrm{Ph}(\mathbf{1 b})$ & $\mathbf{7 b}$ & 8 & 96 & $>95: 5$ & 64 \\
3 & $\mathbf{6 c}$ & $\mathrm{Ph}(\mathbf{1 b})$ & $\mathbf{7 c}$ & 30 & 79 & $87: 13$ & 47 \\
4 & $\mathbf{6 d}$ & $\mathrm{Ph}(\mathbf{1 b})$ & $\mathbf{7 d}$ & 23 & 90 & $94: 6$ & 25 \\
5 & $\mathbf{6 a}$ & $4-\mathrm{MeOC}_{6} \mathrm{H}_{4}(\mathbf{1 a})$ & $\mathbf{7 e}$ & 3 & 96 & $>95: 5$ & 82 \\
6 & $\mathbf{6 a}$ & $4-\mathrm{FC}_{6} \mathrm{H}_{4}(\mathbf{1 d})$ & $\mathbf{7 f}$ & 9 & 91 & $92: 8$ & 70 \\
7 & $\mathbf{6 a}$ & $4-\mathrm{BrC}_{6} \mathrm{H}_{4}(\mathbf{1 e})$ & $\mathbf{7 g}$ & 21 & 84 & $87: 13$ & $61^{\mathrm{e}}$ \\
8 & $\mathbf{6 a}$ & $2-\mathrm{MeC}_{6} \mathrm{H}_{4}(\mathbf{1 f})$ & $\mathbf{7 h}$ & 4 & 96 & $>95: 5$ & 59 \\
\hline
\end{tabular}

${ }^{\text {a }}$ Reaction conditions: 6 ( $\left.0.23 \mathrm{mmol}\right), \mathbf{1}$ (1.1-1.2 equiv), $\mathrm{AgOAc}(3$ mol\%), ligand $(3.3 \mathrm{~mol} \%)$, concentration $(0.12 \mathrm{M}),-25^{\circ} \mathrm{C}$.

${ }^{\mathrm{b}}$ Isolated yields of the anti-isomer based on $\mathbf{6}$.

${ }^{\mathrm{c}} \mathrm{dr}=$ anti/syn, determined by ${ }^{1} \mathrm{H}$ NMR.

${ }^{\mathrm{d}}$ Of the anti-isomer, determined by chiral HPLC analysis.

${ }^{\mathrm{e}}$ The absolute configuration was determined to be $(1 R, 2 R)$ by comparison with literature data. ${ }^{24}$

Our optimization studies began with an investigation into the vinylogous Mannich reaction of aldimines 1a and $\alpha, \alpha-$ dicyanoolefins $6 \mathbf{6}$ catalyzed by silver acetate and $(S)$-SynPhos in diethyl ether at $0{ }^{\circ} \mathrm{C}$. We found that this reaction proceeded readily, gaving the desired vinylogous Mannich adduct in $27 \%$ ee after two hours. In order to further optimize the reaction conditions, we varied solvent, ligands, and reaction temperature, and found the opti- mized conditions for this AVM reaction to be AgOAc, L5, $\mathrm{Et}_{2} \mathrm{O},-25^{\circ} \mathrm{C}$.

As summarized in Table 4, aldimine $\mathbf{1 b}$ reacted smoothly with a variety of $\alpha, \alpha$-dicyanoolefins in good yields and excellent diastereoselectivities, though with moderate enantioselectivities (entries 1-4). ${ }^{26}$ The $\alpha, \alpha$-dicyanoolefin 6a emerged as the best scaffold for the AVM reaction, thus the reactions of $\mathbf{6 a}$ with a series of aldimines bearing various substituents on the aromatic ring were carried out (entries 5-8). This investigation revealed that the enantioselectivity of this AVM was sensitive to both the steric and the electronic properties of the substituents on the phenyl ring of aldimines 1. Generally, phenyl substituents bearing electron-donating groups at the para-position gave a higher ee compared to those with an electron-withdrawing group (entries 5-7). The reaction of $\alpha, \alpha$-dicyanoolefins 6a with aldimine 1a proceeded well and gave the best selectivity ( $82 \%$ ee, dr $>95: 5$, entry 5 ). The presence of an ortho-substituent on the aldimine compromised the enantioselectivity but not the yield or diastereoselectivity (entry 8).

In conclusion, we have developed three asymmetric Mannich-type reactions using an efficient bifunctional silver acetate catalyst. The reactions give high yields, up to $>95: 5$ diastereoselectivities and moderate to excellent enantioselectivities (up to $97 \%$ ee). External base is not required in the bifunctional catalytic procedure, in which acetate promotes the deprotonation of active proton. The special features are the mild reaction conditions and simple operation. This method provides an efficient and convenient route to synthesize optically active $\beta$-amino carbonyl compounds and related compounds.

Further investigations into the capacity of this bifunctional catalyst system toward other catalytic asymmetric Mannich reactions are currently in progress in our laboratory, and results will be reported in due course.

Supporting Information for this article is available online at http://www.thieme-connect.com/ejournals/toc/synlett.

\section{Acknowledgment}

Financial support from the National Natural Science Foundation of China (20621063 \& 20672112) and Dalian Institute of Chemical Physics (K2007F1), Chinese Academy of Sciences are acknowledged.

\section{References and Notes}

(1) (a) Martin, S. F. Acc. Chem. Res. 2002, 35, 895. (b) Córdova, A. Acc. Chem. Res. 2004, 37, 102. (c) Notz, W.; Tanaka, F.; Barbas, C. F. III. Acc. Chem. Res. 2004, 37, 580. (d) Ting, A.; Schaus, S. E. Eur. J. Org. Chem. 2007, 5797. (e) Verkade, J. M. M.; van Hemert, L. J. C.; Quaedflieg, P. J. L. M.; Rutjes, F. P. J. T. Chem. Soc. Rev. 2008, 37, 29.

(2) Viso, A.; de la Pradilla, R. F.; Garcia, A.; Flores, A. Chem. Rev. 2005, 105, 3167. 
(3) For Ag-catalyzed Mannich reactions, see: (a) Ferraris, D.; Young, B.; Dudding, T.; Lectka, T. J. Am. Chem. Soc. 1998, 120, 4548. (b) Josephsohn, N. S.; Snapper, M. L.; Hoveyda, A. H. J. Am. Chem. Soc. 2004, 126, 3734. (c) Josephsohn, N. S.; Carswell, E. L.; Snapper, M. L.; Hoveyda, A. H. Org. Lett. 2005, 7, 2711. (d) Carswell, E. L.; Snapper, M. L.; Hoveyda, A. H. Angew. Chem. Int. Ed. 2006, 45, 7230. (e) Mandai, H.; Mandai, K.; Snapper, M. L.; Hoveyda, A. H. J. Am. Chem. Soc. 2008, 130, 17961. (f) Wieland, L. C.; Vieira, E. M.; Snapper, M. L.; Hoveyda, A. H. J. Am. Chem. Soc. 2009, 131, 570.

(4) For $\mathrm{Cu}$-catalyzed Mannich reactions, see: (a) Nishiwaki, N.; Knudsen, K. R.; Gothelf, K. V.; Jørgensen, K. A. Angew. Chem. Int. Ed. 2001, 40, 2992. (b) Juhl, K.; Gathergood, N.; Jørgensen, K. A. Angew. Chem. Int. Ed. 2001, 40, 2995. (c) Knudsen, K. R.; Risgaard, T.; Nishiwaki, N.; Gothelf, K. V.; Jørgensen, K. A. J. Am. Chem. Soc. 2001, 123, 5843. (d) Kobayashi, S.; Matsubara, R.; Nakamura, Y.; Kitagawa, H.; Sugiura, M. J. Am. Chem. Soc. 2003, 125, 2507. (e) Handa, S.; Gnanadesikan, V.; Matsunaga, S.; Shibasaki, M. J. Am. Chem. Soc. 2007, 129, 4900. (f) Suto, Y.; Kanai, M.; Shibasaki, M. J. Am. Chem. Soc. 2007, 129, 500. (g) Du, Y.; Xu, L. W.; Shimizu, Y.; Oisaki, K.; Kanai, M.; Shibasaki, M. J. Am. Chem. Soc. 2008, 130, 16146.

(5) For Zn-catalyzed Mannich reactions, see: (a) Kobayashi, S.; Hamada, T.; Manabe, K. J. Am. Chem. Soc. 2002, 124, 5640. (b) Matsunaga, S.; Kumagai, N.; Harada, S.; Shibasaki, M. J. Am. Chem. Soc. 2003, 125, 4712. (c) Trost, B. M.; Terrell, L. R. J. Am. Chem. Soc. 2003, 125, 338. (d) Hamada, T.; Manabe, K.; Kodayashi, S. J. Am. Chem. Soc. 2004, 126, 7768. (e) Matsunaga, S.; Yoshida, T.; Morimoto, H.; Kumagai, N.; Shibasaki, M. J. Am. Chem. Soc. 2004, 126, 8777. (f) Yoshida, T.; Morimoto, H.; Kumagai, N.; Matsunaga, S.; Shibasaki, M. Angew. Chem. Int. Ed. 2005, 44, 3470. (g) Trost, B. M.; Jaratjaroonphong, J.; Reutrakul, V. J. Am. Chem. Soc. 2006, 128, 2778.

(6) For Zr-catalyzed Mannich reactions, see: (a) Ishitani, H.; Ueno, M.; Kobayashi, S. J. Am. Chem. Soc. 1997, 119, 7153. (b) Ishitani, H.; Ueno, M.; Kobayashi, S. J. Am. Chem. Soc. 2000, 122, 8180. (c) Ihori, Y.; Yamashita, Y.; Ishitani, H.; Kobayashi, S. J. Am. Chem. Soc. 2005, 127, 15528. (d) Saruhashi, K.; Kobayashi, S. J. Am. Chem. Soc. 2006, $128,11232$.

(7) For other metal-catalyzed Mannich reactions, see: (a) Arend, M. Angew. Chem. Int. Ed. 1999, 38, 2873. (b) Yamada, K.; Harwood, S. J.; Groger, H.; Shibasaki, M. Angew. Chem. Int. Ed. 1999, 38, 3504. (c) Kobayashi, S.; Arai, K.; Shimizu, H.; Ihori, Y.; Ishitani, H.; Yamashita, Y. Angew. Chem. Int. Ed. 2005, 44, 761. (d) Harada, S.;

Handa, S.; Matsunaga, S.; Shibasaki, M. Angew. Chem. Int. Ed. 2005, 44, 4365. (e) Morimoto, H.; Lu, G.; Aoyama, N.; Matsunaga, S.; Shibasaki, M. J. Am. Chem. Soc. 2007, 129, 9588. (f) Cutting, G. A.; Stainforth, N. E.; John, M. P.; Kociok-Köhn, G.; Willis, M. C. J. Am. Chem. Soc. 2007, 129, 10632. (g) Chen, Z.; Morimoto, H.; Matsunaga, S.; Shibasaki, M. J. Am. Chem. Soc. 2008, 130, 2170. (h) Lu, G.; Morimoto, H.; Matsunaga, S.; Shibasaki, M. Angew. Chem. Int. Ed. 2008, 47, 6847. (i) Morimoto, H.; Yoshino, T.; Yukawa, T.; Lu, G.; Matsunaga, S.; Shibasaki, M. Angew. Chem. Int. Ed. 2008, 47, 9125. (j) Xu, Y. J.; Lu, G.; Matsunaga, S.; Shibasaki, M. Angew. Chem. Int. Ed. 2009, 48, 3353. (k) Hamashima, Y.; Sasamoto, N.; Hotta, D.; Somei, H.; Umebayashi, N.; Sodeoka, M. Angew. Chem. Int. Ed. 2005, 44, 1525 .

(8) For proline-catalyzed Mannich reactions, see: (a) List, B. J. Am. Chem. Soc. 2000, 122, 9336. (b) List, B.; Pojarliev, P.; Biller, W. T.; Martin, H. J. J. Am. Chem. Soc. 2002, 124,
827. (c) Córdova, A.; Notz, W.; Zhong, G. F.; Betancort, J. M.; Barbas, C. F. III. J. Am. Chem. Soc. 2002, 124, 1842. (d) Córdova, A.; Watanabe, S.-i.; Tanaka, F.; Notz, W.; Barbas, C. F. III. J. Am. Chem. Soc. 2002, 124, 1866. (e) Hayashi, Y.; Tsuboi, W.; Ashimine, I.; Urushima, T.; Shoji, M.; Sakai, K. Angew. Chem. Int. Ed. 2003, 42, 3677. (f) Hayashi, Y.; Tsuboi, W.; Shoji, M.; Suzuki, N. J. Am. Chem. Soc. 2003, 125, 11208. (g) Zhuang, W.; Saaby, S.; Jørgensen, K. A. Angew. Chem. Int. Ed. 2004, 43, 4476. (h) Ibrahem, I.; Casas, J.; Córdova, A. Angew. Chem. Int. Ed. 2004, 43, 6528. (i) Enders, D.; Grondal, C.; Vrettou, M.; Raabe, G. Angew. Chem. Int. Ed. 2005, 44, 4079.

(j) Marques, M. M. B. Angew. Chem. Int. Ed. 2006, 45, 348. (k) Mitsumori, S.; Zhang, H.; Cheong, P. H. Y.; Houk, K. N.; Tanaka, F.; Barbas, C. F. III. J. Am. Chem. Soc. 2006, 128, 1040. (1) Zhang, H. L.; Mifsud, M.; Tanaka, F.; Barbas, C. F. III. J. Am. Chem. Soc. 2006, 128, 9630. (m) Yang, J. W.; Stadler, M.; List, B. Angew. Chem. Int. Ed. 2007, 46, 609. (n) Gianelli, C.; Sambri, L.; Carlone, A.; Bartoli, G.; Melchiorre, P. Angew. Chem. Int. Ed. 2008, 47, 8700. (o) Hayashi, Y.; Okano, T.; Itoh, T.; Urushima, T.; Ishikawa, H.; Uchimaru, T. Angew. Chem. Int. Ed. 2008, 47, 9053. (p) Zhang, H. L.; Mitsumori, S.; Utsumi, N.; Imai, M.; Garcia-Delgado, N.; Mifsud, M.; Albertshofer, K.; Cheong, P. H. Y.; Houk, K. N.; Tanaka, F.; Barbas, C. F. III. J. Am. Chem. Soc. 2008, 130, 875. (q) Hahn, B. T.; Fröhlich, R.; Harms, K.; Glorius, F. Angew. Chem. Int. Ed. 2008, 47, 9985. (r) Yang, J. W.; Chandler, C.; Stadler, M.; Kampen, D.; List, B. Nature 2008, 452, 453. (s) Chandler, C.; Galzerano, P.; Michrowska, A.; List, B. Angew. Chem. Int. Ed. 2009, 48, 1978.

(9) For Brønsted acid catalyzed Mannich reactions, see: (a) Akiyama, T.; Itoh, J.; Yokota, K.; Fuchibe, K. Angew. Chem. Int. Ed. 2004, 43, 1566. (b) Nugent, B. M.; Yoder, R. A.; Johnston, J. N. J. Am. Chem. Soc. 2004, 126, 3418. (c) Singh, A.; Yoder, R. A.; Shen, B.; Johnston, J. N. J. Am. Chem. Soc. 2007, 129, 3466. (d) Hashimoto, T.; Maruoka, K. J. Am. Chem. Soc. 2007, 129, 10054. (e) Guo, Q. X.; Liu, H.; Guo, C.; Luo, S. W.; Gu, Y.; Gong, L. Z. J. Am. Chem. Soc. 2007, 129, 3790. (f) Yamanaka, M.; Itoh, J.; Fuchibe, K.; Akiyama, T. J. Am. Chem. Soc. 2007, 129, 6756. (g) Sickert, M.; Schneider, C. Angew. Chem. Int. Ed. 2008, 47, 3631. (h) Singh, A.; Johnston, J. N. J. Am. Chem. Soc. 2008, 130, 5866.

(10) For thiurea-catalyzed Mannich reactions, see: (a) Wenzel, A. G.; Jacobsen, E. N. J. Am. Chem. Soc. 2002, 124, 12964. (b) Yoon, T. P.; Jacobsen, E. N. Angew. Chem. Int. Ed. 2005, 44, 466. (c) Taylor, M. S.; Tokunaga, N.; Jacobsen, E. N. Angew. Chem. Int. Ed. 2005, 44, 6700. (d) Zhang, Y.; Liu, Y. K.; Kang, T. R.; Hu, Z. K.; Chen, Y. C. J. Am. Chem. Soc. 2008, 130, 2456. (e) Yalalov, D. A.; Tsogoeva, S. B.; Shubina, T. E.; Martynova, I. M.; Clark, T. Angew. Chem. Int. Ed. 2008, 47, 6624. (f) Wang, C. J.; Dong, X. Q.; Zhang, Z. H.; Xue, Z. Y.; Teng, H. L. J. Am. Chem. Soc. 2008, $130,8606$.

(11) For other organocatalyst-catalyzed Mannich reactions, see: (a) Kano, T.; Yamaguchi, Y.; Tokuda, O.; Maruoka, K. J. Am. Chem. Soc. 2005, 127, 16408. (b) Poulsen, T. B.; Alemparte, C.; Saaby, S.; Bella, M.; Jørgensen, K. A. Angew. Chem. Int. Ed. 2005, 44, 2896. (c) Mizuta, S.; Shibata, N.; Goto, Y.; Furukawa, T.; Nakamura, S.; Toru, T. J. Am. Chem. Soc. 2007, 129, 6394. (d) Ramasastry, S. S. V.; Zhang, H.; Tanaka, F.; Barbas, C. F. III. J. Am. Chem. Soc. 2007, 129, 288. (e) Notte, G. T.; Leighton, J. L. J. Am. Chem. Soc. 2008, 130, 6676. (f) Uraguchi, D.; Koshimoto, K.; Ooi, T. J. Am. Chem. Soc. 2008, 130, 10878. (g) Uraguchi, D.; Ueki, Y.; Ooi, T. J. Am. Chem. Soc. 2008, 
130, 14088. (h) Kano, T.; Yamaguchi, Y.; Maruoka, K. Angew. Chem. Int. Ed. 2009, 48, 1838.

(12) (a) Zeng, W.; Zhou, Y. G. Org. Lett. 2005, 7, 5055. (b) Zeng, W.; Chen, G. Y.; Zhou, Y. G.; Li, Y. X. J. Am. Chem. Soc. 2007, 129, 750. (c) Zeng, W.; Zhou, Y. G. Tetrahedron Lett. 2007, 48, 4619. (d) For a review regarding Ag-catalyzed enantioselective reactions, see: Naodovic, M.; Yamamoto, H. Chem. Rev. 2008, 108, 3132.

(13) For Mannich reactions of imines with $\beta$-dicarbonyl and related compounds, see: (a) Uraguchi, D.; Terada, M. J. Am. Chem. Soc. 2004, 126, 5356. (b) Lou, S.; Taoka, B. M.; Ting, A.; Schaus, S. E. J. Am. Chem. Soc. 2005, 127, 11256. (c) Fini, F.; Bernardi, L.; Herrera, R. P.; Pettersen, D.; Ricci, A.; Sgarzani, V. Adv. Synth. Catal. 2006, 348, 2043. (d) Song, J.; Wang, Y.; Deng, L. J. Am. Chem. Soc. 2006, 128, 6048. (e) Tillman, A. L.; Ye, J. X.; Dixon, D. J. Chem. Commun. 2006, 1191. (f) Ting, A.; Lou, S.; Schaus, S. E. Org. Lett. 2006, 8, 2003. (g) Lou, S.; Dai, P.; Schaus, S. E. J. Org. Chem. 2007, 72, 9998. (h) Marianacci, O.; Micheletti, G.; Bernardi, L.; Fini, F.; Fochi, M.; Pettersen, D.; Sgarzani, V.; Ricci, A. Chem. Eur. J. 2007, 13, 8338. (i) Terada, M.; Tanaka, H.; Sorimachi, K. Synlett 2008, 1661. (j) Hatano, M.; Maki, T.; Moriyama, K.; Arinobe, M.; Ishihara, K. J. Am. Chem. Soc. 2008, 130, 16858.

(k) Yamaoka, Y.; Miyabe, H.; Yasui, Y.; Takemoto, Y. Synthesis 2007, 2571.

(14) Bernardi, L.; Gothelf, A. S.; Hazell, R. G.; Jørgensen, K. A. J. Org. Chem. 2003, 68, 2583.

(15) Ooi, T.; Kameda, M.; Fujii, J.; Maruoka, K. Org. Lett. 2004, 6, 2397.

(16) Okada, A.; Shibuguchi, T.; Ohshima, T.; Masu, H.; Yamaguchi, K.; Shibasaki, M. Angew. Chem. Int. Ed. 2005, 44, 4564.

(17) Kobayashi, S.; Yazaki, R.; Seki, K.; Yamashita, Y. Angew. Chem. Int. Ed. 2008, 47, 5613

(18) Yan, X. X.; Peng, Q.; Li, Q.; Zhang, K.; Yao, J.; Hou, X. L.; Wu, Y. D. J. Am. Chem. Soc. 2008, 130, 14362.

(19) Hernández-Toribio, J.; Arrayás, R. G.; Carretero, J. C. J. Am. Chem. Soc. 2008, 130, 16150.

(20) Shang, D. J.; Liu, Y. L.; Zhou, X.; Liu, X. H.; Feng, X. M. Chem. Eur. J. 2009, 15, 3678.

(21) For asymmetric vinylogous Mannich-type reactions, see: (a) Uraguchi, D.; Sorimachi, K.; Terada, M. J. Am. Chem. Soc. 2004, 126, 11804. (b) Akiyama, T.; Honma, Y.; Itoh,
J.; Fuchibe, K. Adv. Synth. Catal. 2008, 350, 399. (c) Giera, D. S.; Sickert, M.; Schneider, C. Org. Lett. 2008, 10, 4259. (d) González, A. S.; Arrayás, R. G.; Rivero, M. R.; Carretero, J. C. Org. Lett. 2008, 10, 4335. (e) Yamaguchi, A.; Matsunaga, S.; Shibasaki, M. Org. Lett. 2008, 10, 2319.

(22) Liu, T. Y.; Cui, H. L.; Long, J.; Li, B. J.; Wu, Y.; Ding, L. S.; Chen, Y. C. J. Am. Chem. Soc. 2007, 129, 1878.

(23) For vinylogous Michael reactions of $\alpha, \alpha$-dicyanoolefins, see: (a) Xue, D.; Chen, Y. C.; Cun, L. F.; Wang, Q. W.; Zhu, J.; Deng, J. G. Org. Lett. 2005, 7, 5293. (b) Xie, J. W.; Yue, L.; Xue, D.; Ma, X. L.; Chen, Y. C.; Wu, Y.; Zhu, J.; Deng, J. G. Chem. Commun. 2006, 1563. (c) Xie, J. W.; Chen, W.; Li, R.; Zeng, M.; Du, W.; Yue, L.; Chen, Y.C.; Wu, Y.; Zhu, J.; Deng, J. G. Angew. Chem. Int. Ed. 2007, 46, 389.

(24) Niess, B.; Jørgensen, K. A. Chem. Commun. 2007, 1620.

(25) General Procedure for the Silver Acetate Catalyzed Mannich Reactions (Table 4, entry 1): Ligand (0.0075 $\mathrm{mmol})$ and $\mathrm{AgOAc}(1.2 \mathrm{mg}, 0.007 \mathrm{mmol})$ were placed in a dried Schlenk tube under a nitrogen atmosphere and $\mathrm{Et}_{2} \mathrm{O}$ $(1.0 \mathrm{~mL})$ was added. The mixture was stirred at room temperature for about $0.5 \mathrm{~h}$. After cooling to the indicated temperature, $\alpha, \alpha$-dicyanoolefin $(0.23 \mathrm{mmol})$ was added as a solution in $\mathrm{Et}_{2} \mathrm{O}(1.0 \mathrm{~mL})$ followed by addition of aldimine $(0.276 \mathrm{mmol})$. Progress of the Ag-catalyzed Mannich reaction was typically monitored by TLC analysis. Upon consumption of the limiting reagent, the reaction was isolated by a short column of silica gel chromatography directly. The ratio (syn/anti) was determined by NMR spectroscopic analysis of the crude product. The crude adducts were purified by column chromatography on silica gel.

(26) Selected physical and spectral data for 7a: white solid, $64 \%$ ee, $[\alpha]_{\mathrm{D}}{ }^{23}-82.9\left(c 1.03, \mathrm{CHCl}_{3}\right) ; R_{f} 0.55$ (PE-EtOAc, 5:1); HPLC (Chiralpak AD-H column; $i$-PrOH-hexane, 95:5; 0.8 $\left.\mathrm{mL} / \mathrm{min} ; 254 \mathrm{~nm} ; 30^{\circ} \mathrm{C}\right): t_{\mathrm{R}}($ minor $)=8.2 \min , t_{\mathrm{R}}($ major $)=$ $13.6 \mathrm{~min} ;{ }^{1} \mathrm{H}$ NMR $\left(400 \mathrm{MHz}, \mathrm{CDCl}_{3}\right): \delta=8.06(\mathrm{~d}, J=7.9$ $\mathrm{Hz}, 1 \mathrm{H}), 7.51(\mathrm{t}, J=7.4 \mathrm{~Hz}, 1 \mathrm{H}), 7.41-7.24(\mathrm{~m}, 7 \mathrm{H}), 4.94$ $4.92(\mathrm{~m}, 1 \mathrm{H}), 4.53(\mathrm{t}, J=10.4 \mathrm{~Hz}, 1 \mathrm{H}), 3.72-3.68(\mathrm{~m}, 1 \mathrm{H})$, 2.89-2.85 (m, $1 \mathrm{H}), 2.70-2.66(\mathrm{~m}, 1 \mathrm{H}), 1.93-1.89(\mathrm{~m}, 1 \mathrm{H})$, $1.51-1.47$ (m, $1 \mathrm{H}), 1.42(\mathrm{~s}, 9 \mathrm{H}) ;{ }^{13} \mathrm{C}$ NMR $(100 \mathrm{MHz}$, $\left.\mathrm{CDCl}_{3}\right): \delta=175.1,154.7,140.0,138.9,133.5,129.8,129.4$, 128.7, 128.6, 127.0, 126.9, 114.2, 113.6, 82.1, 80.2, 57.5, $49.1,28.3,25.9,25.2$. 